\title{
Resistance and Virulence Patterns in Gram Negative and Gram Positives Rods Isolated from the Hospital Environment in Bucharest, Romania
}

\author{
MIHAELA MAGDALENA MITACHE ${ }^{1,2,7}$, OTILIA BANU33, ELVIRA BORCAN", IRINA GHEORGHE 5,6*, LUMINITA MARUTESCU5,6, \\ MARCELA POPA ${ }^{5,6}$, MIRUNA PREDESCU, ELENA RUSU1,7, ROXANA NEMES ${ }^{1}$, MARIANA CARMEN CHIFIRIUC ${ }^{5,6}$ \\ ${ }^{1}$ Titu Maiorescu University of Bucharest, Faculty of Medicine, 67 Gh. Petrascu Str., 031593, Bucharest, Romania \\ 2Public Health Direction Bucharest, 72-74 Avrig Str., 021578, Bucharest, Romania \\ ${ }^{3}$ IUBCV Prof. Dr C.C.Iliescu, 258 Fundeni Road, 022328, Bucharest, Romania \\ EUniversity of Bucharest, Faculty of Biology, Department of Microbiology, 1-3 Portocalelor Alley, 060101, Bucharest, Romania \\ ${ }^{6}$ Research Institute of the University of Bucharest, Borcea Alley, 040421, Bucharest, Romania \\ Titu Maiorescu University of Bucharest, Faculty of Dental Medicine, 67 Gh. Petrascu Str., 031593, Romania
}

\begin{abstract}
We aimed to investigate the antibiotic resistance and virulence markers in Gram negative bacilli (GNB) and Gram positives coccus (GPC), strains recently isolated from the hospital environmentand from patients with surgical wound infections in order to obtain epidemiologically relevant data. The strains identification was performed with the automated miniApi system. The resistance phenotypes were established using disk diffusion (CLSI, 2017). 61 strains were screened for the production of enzymatic soluble virulence factors: hemolysins, amylase, caseinase, aesculin hydrolysis, DNA-ase, lipase, gelatinase and lecithinase, which give microorganisms the ability to colonize and disseminate in the host. Multiplex PCR reactions were performed for the detection of carbapenemases, aminoglycoside-resistant determinants (AME's), quinolone and tetracycline resistance in GNR and SCCmec cassette type in Staphylococcus aureus strains and to identify the genetic support of cell-associated and soluble virulence factors in E. coli strains (fimH, sfaDE, papC, eaea, cnf1, bfpa, eaf, AggR, EaggE genes) and biofilm production in Acinetobacter baumannii isolates (OmpA). The isolated $E$. coli and $A$. baumannii strains were resistant to â-lactam antibiotics, including penicillins and beta-lactamase inhibitors, third / fourth generation cephalosporins and carbapenems (encoded

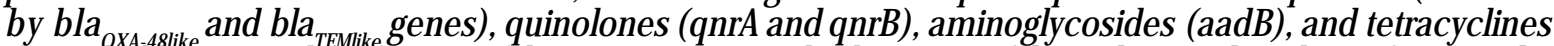
(encoded by tetA and tetB). Most of the strains presented at least two of the eight tested virulence factors. The carbapenemases and ESBLs producers proved to be positive for the majority of the tested soluble virulence factors, proving the pathogenic potential of these strains. In S. aureus isolates the molecular analysis showed that $60 \%$ of the isolates were MRSA and the molecular analysis revealed the presence of the SCCmec cassette type mec IVa and III types. Our data suggest the hypothesis according to which nosocomial origin of the strains can be explained by multiple drug resistance and virulence determinants.
\end{abstract}

Keywords: resistance, virulence, nosocomial infections

Antimicrobial resistance (AMR) represent a growing public health which consist in the capacity of the microorganisms to survive exposure to antibiotic treatment [1]. Infections caused by multidrug resistant (MDR) and virulent Gram-positive and Gram negative bacteria are very common in hospital settings but recently there have been described that are involved also in community environments [2]. The bacteria included in ESKAPE acronym (Enterococcus faecium, Staphylococcus aureus, Klebsiella pneumoniae, Acinetobacter baumannii, Pseudomonas aeruginosa, and Enterobacter spp.) are presently causing most of the nosocomial infections in hospital settings [3-5]. There are several intrinsic factors for e.g. point mutation, gene amplification and extrinsic factors like horizontal transfer of resistant gene by mobile genetic elements such as transposons, integrons or plasmids responsible for the development of AMR. The excessive use of antibiotics is strongly related to the widespread of antibiotic resistant bacteria, especially in Intensive Care Units (ICUs) all over the world, which result in increasing the mortality rates [6]. Several factors affect the risk of nosocomial infections, including underlying disease, severity of illness, length of ICU stay, and usage of invasive devices and procedures.

\section{Experimental part}

Bacterial strains and phenotipic analysis

The study included 61 recently isolated (Sept-Dec 2017) belonging to GNR [ Escherichia coli $(n=15) ;$ A baumannii $(n=20)]$ and GPC [S. aureus $(n=26)]$. The hospital strains were identified using Api 20 E/ Api 20 NE/ API Staff system and confirmed by VITEK2 automatic system.

The antibiotic susceptibility was determined by KirbyBauer standard disk diffusion method [using the antibiotics recommended by CLSI, 2017, 2018 for E. coli us following: meropenem (MEM), imipenem (IMP), ertapenem (ETP), cefazolin (CFZ) cephalotin (CEF) ceftriaxon (CTX), cefpodoxim (CFP) cefuroxime (CXM), cefoxitin (FOX), ceftazidim (CAZ), aztreonam (ATM), cefepime (FEP), amoxicillin-clavulanic acid (AMC), piperacilin-tazobactam (PIP-TZP), ciprofloxacin (CIP), levofloxacin (LEV)

*email: IRYNA_84@yahoo.com; Phone: 0040764341680 
gentamycin (GEN), amikacin (AMK), kanamycin (KAN), nitrofurantoin (NIT), trimethoprim-sulfamethoxazole (SXT), tetracycline (TET), chloramphenicol (CHL) and colistin] and quality control was performed with $E$. coli ATCC 25922, for A. baumannii a reference strain of Pseudomonas aeruginosa - ATCC 27853 and for S. aureus penicillin (PEN), oxacillin (OXA), vancomycin (VAN), gentamycin (GEN), tetracycline (TET), ciprofloxacin (CIP), nitrofurantoin (NIT), trimethoprim-sulfamethoxazole (SXT), chloramphenicol (CHL), rifampin (RIF), linezolid (LZD), clindamycin (CLI) and azithromycin (AZM) using as reference strains $S$. aureus ATCC 25923 and by automated methods (Vitek II).

\section{Evaluation of the soluble enzymatic factors}

The virulence phenotypes were investigated by performing enzymatic tests for the expression of the following soluble virulence factors in overnight culture: haemolysins, DN-ase, pore forming toxins (lecithinase, lipase), proteases (caseinase, gelatinase), amylase and aesculin hydrolysis. Detection of haemolysin production was performed by spotting the fresh cultures on $5 \%$ sheep blood agar medium and incubation at $37^{\circ} \mathrm{C}$ for $24 \mathrm{~h}$. The colourless area around the culture revealed the presence of haemolysis activity. For DNA-ase test, the hydrolysis of DNA in the agar by bacterial DNA-ase activity reduces the agar $\mathrm{pH}$. Positive result if appear a clear zone around growth area. For lipase production the strains were spotted on $1 \%$ Tween 80 agar as a substrate and followed by incubation at $37^{\circ} \mathrm{C}$ for $24 \mathrm{~h}$ and an opaque zone around the spot revealed the positive reaction; for lecithinase production, the cultures were spotted into $2.5 \%$ yolk agar and incubated at $37^{\circ} \mathrm{C}$ for $24 \mathrm{~h}$. A clear zone around the spot indicated the lecithinase production. The protease activity (caseinase and gelatinase) was determined using 15\% soluble casein agar, respectively $3 \%$ gelatine as substrate. The strains were spotted and after incubation at $37^{\circ} \mathrm{C}$ for $24 \mathrm{~h}$, a white precipitate surrounding the growth indicated casein proteolysis, and colourless area around culture due to the gelatin hydrolysis, indicated the positive reaction forgelatinase. Amylase was detected using agar with $1 \%$ starch and hydrolysis was revealed after adding Lugol's solution (yellow ring around the culture, while the rest of the plate will be blue). For the aesculin hydrolysis the medium containing $\mathrm{Fe}^{3+}$ citrate was used and inoculated by spotting, then incubated for $24 \mathrm{~h}$ at $37^{\circ} \mathrm{C}$ temperature. A black precipitate around culture due to esculetol released under the action of beta-galactosidase was considered positive reaction.

\section{Molecular analysis}

Genetic support of ARGs and virulence in GNR

The genetic support of the resistance (carbapenemases, ESBLs, quinolones aminoglycosides and tetracycline's) and virulence in GNR strains (table 1, 2, 3, 4) was investigated by simplex and multiplex PCR, using a reaction mix of 20uL (PCR Master Mix 2x, Thermo Scientific) containing $1 \mu$ l of bacterial DNA extracted using the alkaline extraction method (table 1).

\section{Screening of $S$. aureus resistance and virulence genes by PCR.}

The genotypic characterization of the $\mathrm{SCCmec}$ cassette types present in the analysed strains was performed using PCR methods (simplex and multiplex) in order to elucidate the structure of these genetic elements and to obtain the relevant epidemiological data. Two reactions were performed using the multiplex PCR with five and four pairs of specific primers respectively for the various sequences of the SCCmec cassette. Their classification and parameters used to conduct the reactions followed the protocol developed by Miheirico et al. [21] and Zhang etal. [22]. The detection of the specific virulence genes was performed by three simplex PCR and three multiplex PCR assays according with previous published protocols [23].

The detection of the specific virulence genes was performed by three simplex PCR and three multiplex PCR assays (table 5). The information obtained was used to compare the prevalence of specific resistance and virulence genes amongst nosocomial strains isolated from the hospital settings.

In order to achieve samples in PCR reaction, was used PCR thermal Bio-Rad.

Table 1

THE COMPOSITION OF THE REACTION MIX

\begin{tabular}{|c|c|c|c|c|c|c|c|}
\hline \multirow[t]{2}{*}{ The gene } & \multicolumn{6}{|c|}{ Concentration } & \multirow[t]{2}{*}{ Final volume } \\
\hline & primer & $\mathrm{MgCl}_{2}$ & dNTP & $\begin{array}{c}\text { DNA } \\
\text { Taq-pol }\end{array}$ & $\begin{array}{c}\text { Reaction } \\
\text { buffer }\end{array}$ & DNA & \\
\hline 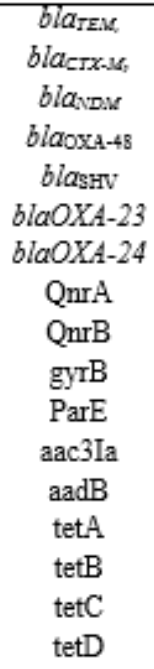 & $0.5 \mu \mathrm{M}$ & $1.2 \mathrm{mM}$ & $2 \mu \mathrm{M}$ & $0.2 \mathrm{U}$ & $1 \mathrm{x}$ & $10 \mathrm{x}$ & $20 \mu \mathrm{l}$ \\
\hline
\end{tabular}


Table 2

PRIMERS SEQUENCES USED IN SIMPLEX AND MULTIPLEX PCR ASSAYS FOR CARBAPENEM RESISTANCE GENES

\begin{tabular}{|c|c|c|c|c|}
\hline The gene & Primer & Nucleotide sequence & $\begin{array}{l}\text { Amplification } \\
\text { size and Tm }\end{array}$ & References \\
\hline \multirow[t]{2}{*}{ bla OXA $-48_{8}$} & OXA-F & GCGTGGTTAAGGATGAACAC & 438 & \multirow[t]{4}{*}[7,8]{} \\
\hline & OXA-R & CATCAAGTTCAACCCAACCG & $52^{\circ} \mathrm{C}$ & \\
\hline \multirow[t]{2}{*}{ bla NDM } & NDM-F & GGTTTGGCGATCTGGTTTTC & 621 & \\
\hline & NDM-R & CGGAATGGCTCATCACGATC & $52^{\circ} \mathrm{C}$ & \\
\hline \multirow[t]{2}{*}{ blaTEM } & TEM-F & ATGAGTTTTCAACATTTTCG & 861 & \multirow[t]{2}{*}{ [9] } \\
\hline & TEM-R & TTACCAATGCTTAATCAG TG & $59^{\circ} \mathrm{C}$ & \\
\hline \multirow[t]{2}{*}{ blashv } & SHV-F & GCCCTCACTCAAGGATGTAT & 888 & \multirow[t]{2}{*}{ [10] } \\
\hline & SHV-R & TTAGCGTTGCCAGTGCTCGA & $58^{\circ} \mathrm{C}$ & \\
\hline \multirow[t]{2}{*}{ bla $a_{\mathrm{CTX}-\mathrm{M}}$} & CTX-M-F & CGCTGTTGTTAGGAAGTGTG & 730 & \multirow[t]{2}{*}{ [11] } \\
\hline & CTX-M-R & GGCTGGGTGAAGTAAGTGAC & $59^{\circ} \mathrm{C}$ & \\
\hline \multirow[t]{2}{*}{ bla $a_{0 x 4-23}$} & OXA-23F & ATGAGTTATCTATTTTGTC & 501 & \multirow[t]{4}{*}{ [12] } \\
\hline & OXA-23R & TGTCAAGCTCTTAAATAATA & $52^{\circ} \mathrm{C}$ & \\
\hline \multirow[t]{2}{*}{ bla $a_{0 x 4-24}$} & OXA-24F & GTACTAATCAAAGTTGTGAA & 246 & \\
\hline & OXA-24R & TTCCCCTAACATGAATTTGT & $52^{\circ} \mathrm{C}$ & \\
\hline
\end{tabular}

Table 3

PRIMERS SEQUENCES USED IN SIMPLEX AND MULTIPLEX PCR ASSAYS FOR QUINOLONE, AMINOGLYCOSIDES AND TETRACYCLINE GENES

\begin{tabular}{|c|c|c|c|c|c|}
\hline QnrAm-F & AGA GGA TTT CTC ACG CCA GG & & \multirow{2}{*}{$60^{\circ} \mathrm{C}$} & \multirow{2}{*}{580} & \multirow{5}{*}{ [13] } \\
\hline$Q n r A m-R$ & TGC CAG GCA CAG ATC TTG AC & & & & \\
\hline$Q n r B m-F$ & GGM ATH GAA ATT CGC CAC TG & $\begin{aligned} \mathrm{M}=\mathrm{A} \text { or } \mathrm{C} \\
\mathrm{H}=\mathrm{A} \text { or } \mathrm{C} \\
\quad \text { or } \mathrm{T}\end{aligned}$ & \multirow{3}{*}{$60^{\circ} \mathrm{C}$} & \multirow[t]{3}{*}{264} & \\
\hline QnrBm-R & TTT GCY GYY CGC CAG TCG AA & $\mathrm{Y}=\mathrm{C}$ or $\mathrm{T}$ & & & \\
\hline QnrSm-R & TCT AAA CCG TCG AGT TCG GCG & & & & \\
\hline$G y r B-F$ & GCGCGAGATGACCCGCCGCA & & \multirow{2}{*}{$60^{\circ} \mathrm{C}$} & & \multirow{4}{*}{ [14] } \\
\hline$G y r B-R$ & CTGGCGGAAGAAGAAGGTCAACA & & & & \\
\hline ParE-F & CGGCGTTCGTCTCGGGCGTGGTGAAGGA & & \multirow{2}{*}{$60^{\circ} \mathrm{C}$} & & \\
\hline Par $E-R$ & TCGAGGGCGTAGTAGATGTCCTTGCCGA & & & & \\
\hline $\begin{array}{l}\text { aac-3-IaF } \\
\text { aac3IAR }\end{array}$ & $\begin{array}{l}\text { ATGGGCATCATTCGCACA } \\
\text { TCTCGGCTTGAACGAATTGT }\end{array}$ & & $59^{\circ} \mathrm{C}$ & & DQ370505 \\
\hline $\begin{array}{l}\text { aadB-F } \\
\text { aadB-R }\end{array}$ & $\begin{array}{l}\text { ATGGACACAACGCAGGTCGC } \\
\text { TTAGGCCGCATATCGCGACC }\end{array}$ & & & 524 & [15] \\
\hline tetA & $\begin{array}{l}\text { GCGCGATCTGGTTCACTCG } \\
\text { AGTCGACAGYRGCGCCGGC }\end{array}$ & & $61^{\circ} \mathrm{C}$ & 164 & \multirow{4}{*}{ [16] } \\
\hline tetB & $\begin{array}{l}\text { TACGTGAATTTATTGCTTCGG } \\
\text { ATACAGCATCCAAAGCGCAC }\end{array}$ & & $61^{\circ} \mathrm{C}$ & 206 & \\
\hline tetC & $\begin{array}{l}\text { GCGGGATATCGTCCATTCCG } \\
\text { GCGTAGAGGATCCACAGGACG }\end{array}$ & & $68^{\circ} \mathrm{C}$ & 207 & \\
\hline tetD & $\begin{array}{l}\text { GGAATATCTCCCGGAAGCGG } \\
\text { CACATTGGACAGTGCCAGCAG }\end{array}$ & & $68^{\circ} \mathrm{C}$ & 187 & \\
\hline
\end{tabular}

Table 4

PRIMERS SEQUENCES USED IN SIMPLEX AND MULTIPLEX PCR ASSAYS FOR VIRULENCE GENES IN GNR ISOLATES

\begin{tabular}{|c|c|c|c|}
\hline The gene & Primer & $\begin{array}{l}\text { Amplification size and } \\
\operatorname{Tm}\end{array}$ & References \\
\hline$\underline{h l y A}$ & $\begin{array}{l}\text { AACAAGGATAAGCACTGT TCTGGC T } \\
\text { ACCATATAAGCGGTCATT CCC GTC A }\end{array}$ & $\begin{array}{l}1,177 \mathrm{bp} \\
60^{\circ} \mathrm{C}\end{array}$ & [18] \\
\hline$s f a D / E$ & $\begin{array}{l}\text { CGGAGGAGTAATTACAAACCTGGCA } \\
\text { CTCCGGAGAACTGGGTG ATCTTA C }\end{array}$ & $\begin{array}{l}408 \mathrm{bp} \\
60^{\circ} \mathrm{C}\end{array}$ & [19] \\
\hline papC2 & $\begin{array}{l}\text { GACGGCTGTACTGCAGGGTGTGGC } \\
\text { ATATCCTTTCTGCAGGGATGCAATA }\end{array}$ & $\begin{array}{l}328 \mathrm{bp} \\
63^{\circ} \mathrm{C}\end{array}$ & [19] \\
\hline fimH & $\begin{array}{l}\text { TGC AGA ACG GAT AAG CCG TGG } \\
\text { GCA GTC ACC TGC CCT CCG GTA }\end{array}$ & $\begin{array}{l}508 \mathrm{bp} \\
63^{\circ} \mathrm{C}\end{array}$ & [17] \\
\hline cnfl & $\begin{array}{l}\text { GAA CTT ATT AAG GAT AGT } \\
\text { CAT TAT TTA TAA CGC TG }\end{array}$ & $\begin{array}{l}543 \mathrm{~kb} \\
40^{\circ} \mathrm{C}\end{array}$ & [20] \\
\hline eaea & $\begin{array}{l}\text { GGCTCAATTTGCTGAGACCACGGTT } \\
\text { GCAAATTTAGGTGCGGGTCAGCGTT }\end{array}$ & $\begin{array}{l}65^{\circ} \mathrm{C} \\
494 \mathrm{bp}\end{array}$ & Designed by Chifiriuc \\
\hline bfpA & $\begin{array}{l}\text { F: CAATGGTGCTTGCGCTTGCT } \\
\text { R: GCCGCTTTATCCAACCTGGT }\end{array}$ & $\begin{array}{l}65^{\circ} \mathrm{C} \\
324 \mathrm{bp}\end{array}$ & Designed by Chifiriuc \\
\hline eaf & $\begin{array}{l}\text { CAGGGTAAAAGAAAGATGATAA } \\
\text { TATGGGGACCATGTAATTATCA }\end{array}$ & $\begin{array}{l}52^{\circ} \mathrm{C}-1 \mathrm{~m} \\
397 \mathrm{bp}\end{array}$ & Designed by Chifiriuc \\
\hline
\end{tabular}


Table 4

CONTINUATED

\begin{tabular}{|c|c|c|c|}
\hline \multirow{2}{*}{ AggR } & $\begin{array}{c}\text { CGATGTATACACAAAAGAAGGA } \\
\text { GCCTAATGAAATATGATGGTACT }\end{array}$ & $\begin{array}{c}56^{\circ} \mathrm{C} \\
640 \mathrm{bp}\end{array}$ & Deeigned by Ch:firiuc \\
\hline \multirow{2}{*}{ EAggE } & $\begin{array}{c}\text { CTGGCGAAAGACTGTATCAT } \\
\text { CAATGTATAGAAATCCGCTGTT }\end{array}$ & $\begin{array}{c}56^{\circ} \mathrm{C} \\
630 \mathrm{bp}\end{array}$ & Designed by Ch.firiuc \\
\hline ompA & $\begin{array}{c}\text { CGCTTCTGCTGGTGCTGAAT } \\
\text { CGTGCAGTACCGTTAGGGTA }\end{array}$ & 531 & \\
\hline epsA & $\begin{array}{l}\text { AGCAACTCCTTATCCAATCC } \\
\text { ACCAGACTCACCCATTACAT }\end{array}$ & 451 & \\
\hline
\end{tabular}

Table 5

NUCLEOTIDE SEQUENCES OF PRIMERS USED IN THE IDENTIFICATION OF THE VIRULENCE GENES

\begin{tabular}{|c|c|c|c|c|c|c|c|c|}
\hline \multirow[t]{2}{*}{ Gene } & \multirow[t]{2}{*}{ Primers } & \multirow[t]{2}{*}{ Nucleotide sequence } & \multicolumn{6}{|c|}{ Amplification program } \\
\hline & & & $\begin{array}{c}\text { Denat } \\
\text { uratio } \\
\mathbf{n}\end{array}$ & $\begin{array}{c}\text { No } \\
\text { cycles }\end{array}$ & $\begin{array}{l}\text { Denaturati } \\
\text { on } \\
\text { in each } \\
\text { cycle }\end{array}$ & Anealing & $\begin{array}{c}\text { Extensi } \\
\text { on }\end{array}$ & $\begin{array}{l}\text { Final } \\
\text { extension }\end{array}$ \\
\hline$b b p$ & $\begin{array}{l}\text { BBP-1 } \\
\text { BBP-2 }\end{array}$ & $\begin{array}{l}\text { AACTACATCTAGTACTCAA } \\
\text { CAACAG } \\
\text { ATGTGCTTGAATAACACCA } \\
\text { TCATCT }\end{array}$ & \multirow{2}{*}{$\begin{array}{l}94^{\circ} \mathrm{C} \\
5 \mathrm{~min}\end{array}$} & \multirow{2}{*}{25} & \multirow{2}{*}{$\begin{array}{c}94^{\circ} \mathrm{C} \\
1 \mathrm{~min}\end{array}$} & \multirow{2}{*}{$\begin{array}{l}55^{\circ} \mathrm{C} \\
1 \mathrm{~min}\end{array}$} & \multirow{2}{*}{$\begin{array}{l}72^{\circ} \mathrm{C} \\
1 \mathrm{~min}\end{array}$} & \multirow{2}{*}{$\begin{array}{l}72^{\circ} \mathrm{C} \\
10 \mathrm{~min}\end{array}$} \\
\hline$e b p S$ & $\begin{array}{l}\text { EBP-1 } \\
\text { EBP-2 }\end{array}$ & $\begin{array}{l}\text { CATCCAGAACCAATCGAAG } \\
\text { AC } \\
\text { CTTAACAGTTACATCATCAT } \\
\text { GTTTATCTTTG }\end{array}$ & & & & & & \\
\hline$f n b B$ & $\begin{array}{l}\text { FNBB-1 } \\
\text { FNBB-2 }\end{array}$ & $\begin{array}{l}\text { GTAACAGCTAATGGTCGAA } \\
\text { TTGATACT } \\
\text { CAAGTTCGATAGGAGTACT } \\
\text { ATGTTC }\end{array}$ & \multirow{4}{*}{$\begin{array}{l}94^{\circ} \mathrm{C} \\
5 \mathrm{~min}\end{array}$} & \multirow{4}{*}{25} & \multirow{4}{*}{$\begin{array}{l}94^{\circ} \mathrm{C} \\
1 \mathrm{~min}\end{array}$} & \multirow{4}{*}{$\begin{array}{l}55^{\circ} \mathrm{C}, \\
1 \mathrm{~min}\end{array}$} & \multirow{4}{*}{$\begin{array}{l}72^{\circ} \mathrm{C} \\
1 \mathrm{~min}\end{array}$} & \multirow{4}{*}{$\begin{array}{l}72^{\circ} \mathrm{C} \\
10 \mathrm{~min}\end{array}$} \\
\hline$f i b$ & $\begin{array}{l}\text { FIB-1 } \\
\text { FIB-2 }\end{array}$ & $\begin{array}{l}\text { CTACAACTACAATTGCCGT } \\
\text { CAACAG } \\
\text { GCTCTTGTAAGACCATTTTC } \\
\text { TTCAC }\end{array}$ & & & & & & \\
\hline$c / f A$ & $\begin{array}{l}\text { CLFA-1 } \\
\text { CLFA-2 }\end{array}$ & $\begin{array}{l}\text { ATTGGCGTGGCTTCAGTGCT } \\
\text { CGTTTCTTCCGTAGTTGCAT } \\
\text { TTG }\end{array}$ & & & & & & \\
\hline$c / f B$ & $\begin{array}{l}\text { CLFB-1 } \\
\text { CLFB-2 }\end{array}$ & $\begin{array}{l}\text { ACATCAGTAATAGTAGGGG } \\
\text { GCAAC } \\
\text { TTCGCACTGTTTGTGTTTGC } \\
\text { AC }\end{array}$ & & & & & & \\
\hline$f n b A$ & $\begin{array}{l}\text { forward } \\
\text { reverse }\end{array}$ & $\begin{array}{l}\text { CACAACCAGCAAATATAG } \\
\text { CTGTGTGGTAATCAATGTC }\end{array}$ & $\begin{array}{l}94^{\circ} \mathrm{C}, \\
5 \mathrm{~min}\end{array}$ & 30 & $\begin{array}{l}94^{\circ} \mathrm{C}, \\
1 \mathrm{~min}\end{array}$ & $\begin{array}{l}50^{\circ} \mathrm{C} \\
1 \mathrm{~min}\end{array}$ & $\begin{array}{l}72^{\circ} \mathrm{C} \\
2 \mathrm{~min}\end{array}$ & $\begin{array}{l}72^{\circ} \mathrm{C} \\
10 \mathrm{~min}\end{array}$ \\
\hline cna & $\begin{array}{l}\text { forward } \\
\text { reverse }\end{array}$ & $\begin{array}{l}\text { AGTGGTTACTAATACTG } \\
\text { CAGGATAGATTGGTTTA }\end{array}$ & $\begin{array}{l}94^{\circ} \mathrm{C} \\
5 \mathrm{~min}\end{array}$ & 30 & $\begin{array}{l}94^{\circ} \mathrm{C} \\
1 \mathrm{~min}\end{array}$ & $\begin{array}{l}55^{\circ} \mathrm{C}, \\
1 \mathrm{~min}\end{array}$ & $\begin{array}{l}72^{\circ} \mathrm{C} \\
2 \mathrm{~min}\end{array}$ & $\begin{array}{l}72^{\circ} \mathrm{C} \\
10 \mathrm{~min}\end{array}$ \\
\hline $\operatorname{coag}$ & $\begin{array}{l}\mathrm{C} 1 \\
\mathrm{C} 2\end{array}$ & $\begin{array}{l}\text { CGAGACCAAGATTCAACAA } \\
\text { G } \\
\text { AAAGAAAACCACTCACATC } \\
\text { AGT }\end{array}$ & $\begin{array}{l}94^{\circ} \mathrm{C}, \\
5 \mathrm{~min}\end{array}$ & 40 & $\begin{array}{l}94^{\circ} \mathrm{C}, \\
30 \mathrm{sec}\end{array}$ & $\begin{array}{l}55^{\circ} \mathrm{C} \\
30 \text { sec. }\end{array}$ & $\begin{array}{l}72^{\circ} \mathrm{C} \\
1,5 \mathrm{~min}\end{array}$ & $\begin{array}{l}72^{\circ} \mathrm{C} \\
5 \mathrm{~min}\end{array}$ \\
\hline $\begin{array}{l}\text { lukk- } \\
P V\end{array}$ & $\begin{array}{l}\text { lukk-PV-1 } \\
\text { luk-PV-2 }\end{array}$ & $\begin{array}{l}\text { ATCATTAGGTAAAATGTCT } \\
\text { GGACATGATCCA } \\
\text { GCATCAASTGTATTGGATA } \\
\text { GCAAAAGC }\end{array}$ & \multirow{2}{*}{$\begin{array}{l}95^{\circ} \mathrm{C} \\
5 \mathrm{~min}\end{array}$} & \multirow{2}{*}{30} & \multirow{2}{*}{$\begin{array}{l}94^{\circ} \mathrm{C} \\
30 \mathrm{sec}\end{array}$} & \multirow{2}{*}{$\begin{array}{l}55^{\circ} \mathrm{C} \\
2 \mathrm{~min}\end{array}$} & \multirow{2}{*}{$\begin{array}{l}72^{\circ} \mathrm{C} \\
1 \mathrm{~min}\end{array}$} & \multirow{2}{*}{$\begin{array}{l}72^{\circ} \mathrm{C} \\
10 \mathrm{~min}\end{array}$} \\
\hline hig & $\begin{array}{l}\text { hlgC } \\
\text { hlgB }\end{array}$ & $\begin{array}{l}\text { GCCAATCCGTTATTAGAAA } \\
\text { ATGC } \\
\text { CCATAGACGTAGCAACGGA } \\
\text { T }\end{array}$ & & & & & & \\
\hline tst & $\begin{array}{l}\text { tst1 } \\
\text { tst2 }\end{array}$ & $\begin{array}{l}\text { CATCTACAAACGATAATAT } \\
\text { AAAGG } \\
\text { CATTGTTATTTTCCAATAAC } \\
\text { CACCCG }\end{array}$ & $\begin{array}{l}94^{\circ} \mathrm{C}, \\
5 \mathrm{~min}\end{array}$ & 30 & $\begin{array}{l}94^{\circ} \mathrm{C}, \\
30 \mathrm{sec}\end{array}$ & $\begin{array}{l}58^{\circ} \mathrm{C} \\
30 \mathrm{sec}\end{array}$ & $\begin{array}{l}72^{\circ} \mathrm{C} \\
2 \mathrm{~min}\end{array}$ & $\begin{array}{l}72^{\circ} \mathrm{C}, \\
5 \mathrm{~min}\end{array}$ \\
\hline
\end{tabular}

\section{Results and discussions}

Phenotypic results of the distribution of resistance profiles in analysed $E$. coli isolates have shown that the majority of the strains were resistant to IMP, CAZ and FEP
(73.33\%), followed by MEM and AMK (66.66\%). $53.33 \%$ of the strains were resistant to cephalosporin's (CTX, CXM), PIP-TZP and CIP. A low percentage of $E$. coli strains were resistant to ATM and ETP (20\%) (fig. 1). A. baumannii antibiotic resistance profiles revealed a high level of 
Antibiotic resistance profiles (\%) in E.coli, A. baumannii and $S$. aureus isolates

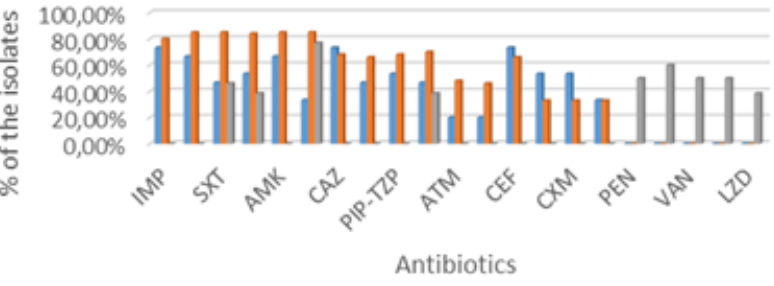

E. coli $=$ A. baumannii $=$ S. aureus

Fig. 1. Graphic representation of antibiotic resistance profile in analysed strains

resistance to MEM, AMK, GEN and SXT ( $85 \%$ of the isolates); quinolones (84\% were resistant to CIP and LEV) followed by IMP ( $80 \%)$, TET (70\%) and CAZ (68\%). The lowest level of resistance was founded to cephalosporin's (fig. 1).

The distribution of carbapenemases in GNR rods have shown the presence of carbapenemases OXA-48 ( $70 \%$ of the strains) and TEM (10\%) in E. coli (); OXA-23 and OXA24 in $A$. baumannii strains (53\% respectively $47 \%$ ). Previously, Bonin et al., in 2011 revealed the co-expression of bla $x_{\text {XX.23 }}$ and bla $a_{\text {pe }}$ in A. baumannii isolated from patients in Timisoara, Arad and Resita [24]. More recently Gheorghe etal., in 2015 demonstrated the presence of bla ${ }^{2}$ within Tn2008 truncating a TnaphA6 transposon and related to CC92 clones (ST437, ST764 and ST765) in A. baumannii strains isolated from Fundeni and Prof. C.C. Iliescu in Bucharest, Romania [25]. In 2016 Georgescu et al., demonstrated first time the presence of one variant of bla $a_{0 \times-24 / 4}$ in $A$. baumannii (bla $a_{0 \times-72}$ ) isolated from from chronic leg ulcer samples [26]. In 2017 Dahdouh et al., revealed also a high prevalence of blaOXA-24 with an increased of virulence factors production in carbapenem resistant Acinetobacter baumannii (CRAB) isolated between 2009 and 2013 from a Spanish hospital (Madrid) [27]. Similar to our results, Pournaras et al., in 2017 have shown that the OXA-58 was the predominant carbapenemase revealed among CRAB isolated from 11 hospitals between 2000 and 2009 in Greece [28].

AME's were demonstrated by the presence of acetyltransferases in A. baunmannii strains ( $10 \%$ were positives for aadB gene).

Among the quinolone resistant clinical strains the plasmid-mediated quinolone resistance qnrB gene was identified in $20 \%$ of E.coli isolates harbouring bla ${ }_{0 \times A-48}$ and qnrA in $15 \%$ of $E$. coli strains.

All tetracycline resistant GNR isolates were positives for tetA and tetB genes. Regarding antibiotic resistance profiles in $\mathrm{S}$. aureus isolates, a high percentage were resistant to GEN (76.92\%); 60\% to OXA, 50\% of the investigated strains were resistant to PEN and CLI and closer percentages of both analysed species were resistant to SXT (46.66\%/46.15\%), and $38.46 \%$ to CIP, TET and LZD (fig. 1).

The phenotypic analysis of the resistance patterns showed that $60 \%$ of $S$. aureus strains revealed the MRSA phenotype from which $80 \%$ were positive for mecA gene. Another study have demonstrated a very close percentages regarding MRSA isolated from patients hospitalized in the same place between 2011- 2014 [29].

Regarding the SCC type the molecular analysis through PCR arrays showed that $40 \%$ of the isolates belonged to SCC mec Type IV with subtypes IVa and $40 \%$ to SCCmecType III. Previous study of our research team have demonstrated the presence of SCCmectype II, V, IIIA and IVB in MRSA isolates recovered from hospital surfaces after decontamination with quaternary ammonium compounds, triclosan and iodine disinfectants in Public Health Diagnostic and Research Laboratory, Bucharest [30].

The distribution of virulence factors of the analysed strains has revealed that the majority $(86.88 \%)$ were positive for the production of lipase, followed by lecithinase

Table 6

THE ENZYMATIC VIRULENCE FACTORS AMONG THE ANALYSED STRAINS (“- "INDICATES THE ABSENCE OF THE VIRULENCE FACTOR," + "THE PRESENCE OF THE VIRULENCE FACTORS ANALYSED)

\begin{tabular}{|c|c|c|c|c|c|c|c|c|}
\hline $\begin{array}{c}\text { Strain } \\
\text { Code/no }\end{array}$ & $\begin{array}{l}\text { Aesculin } \\
\text { hydrolisis }\end{array}$ & DN-ase & Lipase & Caseinase & Lecithinase & Gelatinase & Amylase & Hemolysin \\
\hline 1 E.coli & + & + & + & + & + & - & + & $\alpha$ \\
\hline 2 E.coli & + & - & + & + & + & - & + & $\gamma$ \\
\hline 3 E.coli & - & - & + & - & + & - & + & $\beta$ \\
\hline 4 E.coli & + & - & + & + & + & - & + & $\alpha$ \\
\hline 5 E.coli & - & - & + & - & - & - & + & $\beta$ \\
\hline 6 E.coli & + & - & - & + & + & - & - & $\beta$ \\
\hline 7 E.coli & + & + & + & + & + & - & - & $\alpha$ \\
\hline 8 E.coli & - & - & + & + & + & - & - & $\alpha$ \\
\hline 9 E.coli & + & - & + & - & + & - & - & $\gamma$ \\
\hline 10 E.coli & - & - & + & + & + & - & - & $\beta$ \\
\hline 11 E.coli & + & - & + & + & + & - & - & $\gamma$ \\
\hline 12 E.coli & + & + & + & - & + & - & - & $\alpha$ \\
\hline 13 E.coli & - & - & + & + & - & - & - & $\alpha$ \\
\hline 14 E.coli & + & - & + & + & + & - & - & $\alpha$ \\
\hline 15 E.coli & + & - & + & + & + & - & - & $\alpha$ \\
\hline $16 A b c$ & - & & + & + & + & - & + & $\alpha$ \\
\hline $17 A b c$ & - & - & + & + & - & - & + & $\alpha$ \\
\hline $18 A b c$ & - & - & + & + & - & - & + & $\alpha$ \\
\hline $19 A b c$ & - & - & + & + & - & - & + & $\gamma$ \\
\hline
\end{tabular}


Table 6

CONTINUATED

\begin{tabular}{|c|c|c|c|c|c|c|c|c|}
\hline $20 A b c$ & - & + & + & - & - & - & + & $\gamma$ \\
\hline $21 A b c$ & - & + & + & - & - & - & + & $\gamma$ \\
\hline $22 A b c$ & - & - & + & - & - & - & + & $\gamma$ \\
\hline $23 A b c$ & + & - & + & - & - & - & + & $\alpha$ \\
\hline $24 A b c$ & + & - & + & - & - & - & + & $\alpha$ \\
\hline $25 A b c$ & + & - & + & - & - & - & + & $\alpha$ \\
\hline $26 A b c$ & - & + & + & - & - & - & - & $\gamma$ \\
\hline $27 A b c$ & - & + & + & - & - & - & - & $\gamma$ \\
\hline $28 \mathrm{Abc}$ & - & - & - & - & - & - & - & $\gamma$ \\
\hline $29 A b c$ & - & - & - & - & - & - & - & $\alpha$ \\
\hline $30 A b c$ & - & + & - & - & - & - & - & $\alpha$ \\
\hline $31 A b c$ & - & + & + & - & - & - & - & $\alpha$ \\
\hline $32 A b c$ & - & + & + & - & - & - & - & $\alpha$ \\
\hline $33 A b c$ & - & - & + & - & - & - & - & $\alpha$ \\
\hline $34 A b c$ & - & - & + & - & - & - & - & $\alpha$ \\
\hline $35 A b c$ & - & + & + & - & - & - & - & $\gamma$ \\
\hline 36 S. aureus & + & + & + & - & + & + & - & $\gamma$ \\
\hline 37 S. aureus & - & + & + & + & + & + & - & $\alpha$ \\
\hline 38 S. aureus & + & - & + & - & + & + & - & $\gamma$ \\
\hline 39 S. aureus & + & + & + & + & + & + & - & $\gamma$ \\
\hline 40 S. aureus & - & + & - & + & + & - & - & $\alpha$ \\
\hline 41 S. aureus & + & + & - & + & - & - & - & $\gamma$ \\
\hline 42 S. aureus & + & - & + & + & + & + & + & $\beta$ \\
\hline 43 S. aureus & + & - & + & - & + & + & + & $\beta$ \\
\hline 44 S. aureus & - & + & + & + & + & - & - & $\alpha$ \\
\hline 45 S. aureus & + & + & + & + & + & + & - & $\alpha$ \\
\hline 46 S. aureus & + & + & + & - & - & + & - & $\alpha$ \\
\hline 47 S. aureus & + & - & + & - & + & - & - & $\beta$ \\
\hline 48 S. aureus & + & - & + & + & + & - & + & $\beta$ \\
\hline 49 S. aureus & + & + & - & - & + & + & + & $\alpha$ \\
\hline 50 S. aureus & + & - & + & + & + & - & - & $\beta$ \\
\hline 51 S. aureus & + & - & - & - & + & - & - & $\beta$ \\
\hline 52 S. aureus & + & + & + & + & + & - & - & $\gamma$ \\
\hline 53 S. aureus & + & + & + & + & + & + & - & $\alpha$ \\
\hline 54 S. aureus & + & + & + & - & + & - & - & $\gamma$ \\
\hline 55 S. aureus & + & + & + & - & + & + & + & $\gamma$ \\
\hline 56 S. aureus & + & + & + & + & + & + & - & $\gamma$ \\
\hline 57 S. aureus & + & - & + & + & + & - & + & $\alpha$ \\
\hline 58 S. aureus & - & - & + & - & + & - & - & $\gamma$ \\
\hline 59 S. aureus & + & - & + & - & + & - & - & $\beta$ \\
\hline 60 S. aureus & + & - & + & + & + & - & - & $\gamma$ \\
\hline 61S. aureus & + & + & + & - & + & - & - & $\alpha$ \\
\hline
\end{tabular}

an enzyme involved in dissemination of the infections, (62.29\% of the isolates) aesculin hydrolysis (57.37\%), caseinase - a protease that contribute to tissue degradation (47.54\%) and DN-ase (40.98\%) (table 6). A low percentage of investigated strains produced gelatinase which revealed the production of proteases with large-spectrum proteolytic activity. Similar to this study, Gheorghe et al., in 2017 revealed a high percentage of $S$. aureus isolated from acneiform reactions pustule and periungual lesions were positive for lecithinase, lipase and caseinase [31].
E. coli virulence factors are represented by adhesins [ $P$ fimbriae (papG), type 1 fimbriae (fimH), S fimbriae (sfa) and A fimbriae (afa)]; toxins, such as hemolysin A (hlyA) and cytotoxic necrotizing factor 1 (cnf1); iron uptake, such as aerobactin (aer); protectins, such as serum resistance (traT); and others, such as pathogenicity-associated islands (PAIs) and Tir-containing protein of E. coli (tcpc) [32-34]. Regarding virulence markers in $E$. coli isolates our study revealed the presence of fimH gene ( $66 \%$ of the strains); sfaDE (46.66\%) and cnf1 (33.33\%). In Romania, Mladin et 
al., in 2009 revealed the presence of fimH (in high percentage) and cnfl genes in E. coli nosocomial strains isolated from the Neuropsychiatry Clinical Hospital of Craiova, during December 2006 - November 2007 [35].

Opposite to us, Chelariu et al., in 2017 demonstrated a low percentage of this virulence markers in Enterobacteriaceae strains isolated from stool samples in patients with metabolic syndrome [36]. Mitache et al., revealed the co-expression of several virulence markers: fimH, papC, sfaDE and cnf1 in Enterobacteriaceae isolated from hospital surfaces after decontamination with quaternary ammonium compounds, triclosan and iodine disinfectants in Public Health Diagnostic and Research Laboratory from Bucharest in 2017 [30].

In $A$. baumannii, the investigated isolates are equipped with not only enzymatic resistance mechanisms, but also the ompA biofilm-producing virulence factor $(66.66 \%$ of the analysed strains). Similar to our study, Handal et al., in 2017 have been demonstrated a high percentages of $A$. baumannii isolates positive for OmpA gene [15].

The molecular analysis of selected virulence genes in $S$. aureus isolates showed that $40 \%$ were positives for clfA gene, $35 \%$ for clfB gene and a lower percentages for fib and hlg gene ( $15 \%$ and $7 \%$ respectively). These results regarding the presence of the clfA, clfB, fib and hlg genes highlight the importance of the adherence stage in the development of the invasive infections determined by $S$. aureus regardless of the infectious sources. Very closer percentages were demonstrated by Gheorghe et al., in 2017 in S. aureus strains isolated in 2016 from acneiform reactions pustule and periungual lesions in patients with cutaneous drug adverse reactions in Bucharest, Romania [31].

\section{Conclusions}

The obtained data revealed that the isolated strains harbour multiple drug resistance and virulence determinants, raising the need for the implementation of screening and intervention measures for the prevention of infections with MDR and virulent strains occurred in hospitalized patients.

Acknowledgments: The financial support of the research grant for young researchers no. 27/2017 (28542) awarded by ICUB is gratefully acknowledged.

\section{References}

1. RAGHUNATH D. J. Biosci., 33, No. 4, 2008, p. 593-603.

2. PELEG A., HOOPER D. N. Engl. J. Med., 362 No. 19, 2010, p. 18041813.

3. RICE LB. J. Infect. Dis., 197, 2008, p. 1079-81.

4. CURUTIU C., CHIFIRIUC M.C., MITACHE M.M. Current Organic Chem. 17 No. 2, 2013, p. 149-154(6).

5. MITACHE, M.M., CURUTIU, C., RUSU, E., BAHNA, R., DITU, M., MOLDOVAN, H., HANCU, V., CHIFIRIUC, M.C. Rev. Chim. (Bucharest), 68, no. 3, 2017, p. 566

6. SYDNOR, E.R.M., PERL, T.M. Clin. Microbiol. Review, 2011, p. 141173.

7. POIREL L., WALSH T.R., CUVILLIER V., NORDMANN P. Diagnostic Microbiol and Infect Dis. 70 No. 1, 2011, p. 119-123.

8. JIANG X., NI Y., JIANG Y., YUAN F., HAN L., LI M., et al. J. Clin. Microbiol, 43, 2005, p. 826-831.

9. EFREKAR R.F., HOSSEINI-MAZINANI S.M., GHANDILI S., HAMRAZ M., ZAMANI S. Iranian J Biotech, 3, No. 1, 2005, p. 48-54.

10. NAAS T., PHILIPPON L., POIREL L., RONCO E., NORDMANN P. Antimicrob Agents Chemother, 43, 1999, p. 1281-4.
11. ISRAIL A., CHIFIRIUC C., PALADE G., COTAR A. Clinical and bacteriological aspects of bacterial infections associated to abdominal surgical emergencies. Ars Docenti Publ. House, 2013, p.150.

12. WOODFORD N., ELLINGTON M.J., COELHO J.M., TURTON J.F., WARD M.E., BROWN S., et al. Int. J. Antimicrob. Agents., 27, No. 4, 2006, p. 351-3.

13. CATTOIR V., POIREL L., ROTIMI V., SOUSSY C.J ., NORDMANN P. J. Antimicrob. Chemother., 60, No. 2, 2007, p. 394-7.

14. AGNELLO M., WONG-BERINGER A. PLoS One, 7, No. 8, 2012, e42973.

15. HANDAL R., QUNIBI L., SAHOURI I., JUHARI M.,DAWODI R., MARZOUQA H., HINDIYEH M. Int. Jour. of Microbiol., Article ID 8012104, 2017, p. 7

16. AMINOV R.I., CHEE-SANFORD J.C., GARRIGUES N., TEFEREDEGNE B., KRAPAC I.J., WHITE B.A., MACKIE R.I. Appl. Environ. Microbiol., 68, 2002, p. 1786-1793.

17. JOHNSON J.R., KUSKOWSKI M.A., GAJEWSKI A. et al. J. infect. Dis., 191, 2005, p. 46-50.

18. YAMAMOTO S., TERAI A., YURI K. et al. FEMS Immunol. Med. Microbiol., 12, 1995, p. 85-90.

19. LE BOUGUENEC C., ARChAMBAUd M., LABIGNE A. J. Clin. Microbiol., 30, 1992, p. 1189-1193.

20. BLANCO M., BLANCO J.E., ALONSO M.P. et al. Res. Microbiol., 148, 1997, p. 745-755.

21. MIHEIRICO C., OLIVEIRA D.C., DE LENCASTRE H. J. Antimicrob. Chemother., 51, No. 9, 2007, p. 3374-3377.

22. ZHANG K., MCCLURE J.A., ELSAYED S., LOUIE T., CONLY J. J. Clin. Microbiol., 43, No. 10, 2005, p. 5026-33.

23. COTAR A.I., CHIFIRIUC M.C., DINU S., BUCUR M., IORDACHE C., BANU, O., DRACEA O., LARION C., LAZAR V. Int. J. Mol. Sci., 11, No. 12, 2010, p. 5273-5291.

24. BONIN R.A., POIREL L., LICKER M., NORDMANN P. Clin. Microbiol. Infect., 17, No. 10, 2011, p. 1524-1527.

25. GHEORGHE I., NOVAIS A., GROSSO F., RODRIGUES C., CHIFIRIUC M.C., LAZAR V., PEIXE L. J. Antimicrob. Chemother., 70, No. 4, 2015, p. 1016-1020.

26. GEORGESCU M., GHEORGHE I., DUDU A., CZOBOR I., COSTACHE M, CRISTEA V.C., LAZAR V., CHIFIRIUC M.C. New Microbes and New Infections, 13; 2016, p.87-88.

27. DAHDOUH E., GOAMEZ-GIL R., PACHO S., MINGORANCE J ., DAOUD Z., SUAAREZ M. Plos One, 2017, https://doi.org/10.1371/ journal.pone.0176824.

28. POURNARAS S., DAFOPOULOU K., DEL FRANCO M., ZARKOTOU O., DIMITROULIA E., PROTONOTARIOU E., POULOU A., ZARRILLI R., TSAKRIS A. On behalf of the Greek Study Group on Acinetobacter Antimicrobial Resistance. Int. J. Antimicrob. Agents, 49 No.6, 2017, p. 749-753

29. IONESCU B., IONESCU D., GHEORGHE I., CURUIU C., BANU O., BLEOTU C., MIHAESCU G., LAZAR V., GRIGORE R., BEZIRTZOGLOU E., BERTE'TEANU S. Rom. Biotechnol. Lett., 20, 2014, p. 10536-46.

30. MITACHE, M.M., GHEORGHE, I., TOTEA, G., BLEOTU, C., CURUTIU, C., COCHIOR, D., RUSU, E., CHIFIRIUC ,M.C. Rev. Chim. (Bucharest), 68, no. 5, 2017, p. 2533-2537

31.GHEORGHE I., TATU A.L. , LUPU I., THAMER O., COTAR A.I., PIRCALABIORU G., POPA M., CRISTEA V.C., LAZAR V., CHIFIRIUC M.C. Rom. Biotech. Lett., 22, 1, 2017.

32. JOHNSON J.R., RUSSO T.A. J. Lab. Clin. Med., 139, 2002, p. 155162.

33. CIRL C., WIESER A., YADAV M., DUERR S., SCHUBERT S., FISCHER H., et al. Nat. Med., 14, No. 4, 2008, 399-406.

34. YADAV M., ZHANG J., FISCHER H., HUANG W., LUTAY N., CIRL C., et al. PLoS Pathog, 6, No. 9, 2010, e1001120.

35. MLADIN C., USEIN C.R., CHIFIRIUC M.C., PALADE A., SLAVU C.L., NEGUT M., DAMIAN M. Rom. Biotech. Lett., 2009, 14 (6) p. 4900-4905. 36. CHELARIU M., GROSU M., GHEORGHE I., GRADISTEANU G., PICU A., PETCU L., POPA M., CHIFIRIUC M.C., DITU L.M., LAZAR V., DIMOV T.V. Rom Biotechnol Lett, 22, No. 3. 2017, p. 12643- 12650. 